\title{
Hidden attractors from the switching linear systems
}

\author{
F. Delgado-Aranda ${ }^{a, *}$, I. Campos-Cantón ${ }^{a}$, E. Tristán-Hernández ${ }^{a}$, and P. Salas-Castro ${ }^{b}$ \\ ${ }^{a}$ Instituto de Investigación en Comunicación Óptica, Universidad Autónoma de San Luis Potosí, \\ Álvaro Obregón 64, 78000 San Luis Potosí, S.L.P., México, \\ *e-mail: fdelgadoaranda@gmail.com \\ ${ }^{b}$ Universidad Politécnica de San Luis Potosí, \\ Urbano Villalón 500, 78363 San Luis Potosí, S.L.P., México.
}

Received 14 January 2020; accepted 21 May 2020

\begin{abstract}
Recently, chaotic behavior has been studied in dynamical systems that generate hidden attractors. Most of these systems have quadratic nonlinearities. This paper introduces a new methodology to develop a family of three-dimensional hidden attractors from the switching of linear systems. This methodology allows to obtain strange attractors with only one stable equilibrium, attractors with an infinite number of equilibria or attractors without equilibrium. The main matrix and the augmented matrix of every linear system are considered in RouchéFrobenius theorem to analyze the equilibrium of the switching systems. Also, a systematic search assisted by a computer is used to find the chaotic behavior. The basic chaotic properties of the attractors are verified by the Lyapunov exponents.
\end{abstract}

Keywords: Chaos; hidden attractor; equilibrium; linear system

PACS: 02.10.Ud; 02.10.Yn; 05.45.Pq; 82.40.Bj

DOI: https://doi.org/10.31349/RevMexFis.66.683

\section{Introduction}

The first chaotic system was introduced by Edward Lorenz [1]. From this, the interest in developing new similar systems emerged, and it has been applied in several applications such as securing communications [2], synchronization of systems [3], power systems [4], among others. In dynamical systems, equilibrium is an important characteristic, since the motion of trajectories depend on the local behavior around the neighborhood of the fixed point. Most of the chaotic systems reported in the literature have a determined number of equilibria, and these have been widely studied. For instance, the Lorenz [1], Chen [5], Lü [6] and Chua [7] systems have three equilibrium points, and the Rössler system [8] has only two equilibrium points. Likewise, Sprott [9] has proposed a set of chaotic systems where some of them have only one or two equilibrium points. Moreover, some chaotic systems have an equilibrium point in each basin of attraction which is generated by a piecewise linear function (PWL) [10-12]. The PWL function allows to obtain a set of linear systems, each one having a hyperbolic equilibrium point. These systems can generate three or more scrolls in an attractor; thus, they are known as multi scroll attractors.

Similar systems have been studied with two or more positive Lyapunov exponents. According to [13], these dynamical systems are called hyperchaotic.

In recent years, some systems that generate chaotic attractors which their basin of attraction does not intersect with its neighborhoods of the equilibrium points have been found, these are known as hidden attractors. Hidden attractors are difficult to find because both basins of attraction and the dimension of the attractor could be very small. According to [14], the systems with hidden attractors are classified as a hidden attractor without equilibrium, a hidden attractor with an infinite number of equilibria, and a hidden attractor with one stable equilibrium. For these systems, it is not possible to apply the Shilnikov theorem $[15,16]$ to demonstrate the existence of chaos, because they do not have homoclinic and heteroclinic orbits.

From Sprott's A-system [9], chaotic systems without equilibrium such as Pham [17], C. Wang [18], Azar [19], Wei [20] and Jafari [21] systems have been developed. Also, hyperchaotic systems [22], multi scroll systems [23-25], and fractional-order systems $[26,27]$ without equilibrium have been studied.

On the other hand, some authors have developed chaotic systems that present an infinite number of equilibria. For instance, Jafari [28], Chumbiao [29] and Li [30] systems. Besides it has been found that some hyperchaotic systems [31] and fractal-order systems [32] has a line of equilibria.

Finally, the systems with only one stable equilibrium have been studied from the Sprott's E-system. Some authors, such as Wang [33], Pham [34], among others, proposed similar systems through quadratic nonlinearities. Also, systems with two stable equilibria $[35,36]$ have been developed, and this opens the possibility to design multi scroll systems in the future.

Notice that the chaotic systems mentioned above present quadratic nonlinearities. However, there are a few systems in the literature that generate hidden attractors from switching linear systems $[37,38]$. In these researches, the main methodology is to find hidden degeneracies in piecewise smooth dynamical systems through the sliding mode methods, and they use of efficient analytical-numerical methods for the study of hidden attractors. The approach is based on the use of modern computers and the development of numerical methods. Chua's system was used to find hidden attractors trough these approaches. Due to this motivation, this work presents a new methodology to develop a family of three-dimensional 
hidden attractors from the switching linear systems. The Rouché-Frobenius theorem is used as a tool to analyze the equilibrium of every switching system. Thus, these systems can generate a chaotic system without equilibrium, a chaotic system with an infinite number of equilibrium points, or a chaotic with a stable equilibrium.

This paper is organized as follows: In Sec. 2, the preliminary theory of linear systems is introduced, and its relationship with the equilibrium of the dynamical system. The switched systems without equilibrium, with an infinite number of equilibria, and with stable equilibrium are introduced in Secs. 3 to 5. Finally, a discussion of the proposed systems and the conclusions are given in Secs. 6 and 7, respectively.

\section{Equilibrium description of a linear system}

This section contains briefly the study of the equilibrium point of a linear system, and its main features are presented. Consider a three-dimensional linear dynamical system given by

$$
\dot{x}=A x+B,
$$

where $x=\left[x_{1}, x_{2}, x_{3}\right]^{T} \in \mathbb{R}^{3}$ is the state variable, $B=$ $\left[b_{1}, b_{2}, b_{3}\right]^{T} \in \mathbb{R}^{3}$ stands for a vector of real constants and $A \in \mathbb{R}^{3 \times 3}$ is a linear operator given by

$$
A=\left(\begin{array}{ccc}
a_{11} & a_{12} & a_{13} \\
a_{21} & a_{22} & a_{23} \\
a_{31} & a_{32} & a_{33}
\end{array}\right) .
$$

Assume the system (1) is dissipative; that means, the sum of its eigenvalues is negative, and it has stable $\left(W^{S}\right)$ and unstable $\left(W^{U}\right)$ manifolds. The behavior of the system is defined through the eigenvalues of $A$, which are obtained from the characteristic polynomial

$$
g(\lambda)=\lambda^{3}-\beta \lambda^{2}+\gamma \lambda-\delta,
$$

where $\beta=a_{11}+a_{22}+a_{33}, \gamma=a_{11} a_{22}+a_{11} a_{33}+a_{22} a_{33}-$ $a_{12} a_{21}-a_{13} a_{31}-a_{23} a_{32}$ and $\delta=\operatorname{det}(A)$. The eigenvalues of the system are determined through the discriminant of the polynomial $g(\lambda)$. By definition, the discriminant of a thirddegree polynomial is as follows

$$
\Delta=18 \beta \gamma \delta-4 \beta^{3} \delta+\beta^{2} \gamma^{2}-4 \gamma^{3}-27 \delta^{2},
$$

where the next cases are considered: (1) if $\Delta>0$, the dynamical system (1) has three different eigenvalues; (2) if $\Delta=0$, the dynamical system (1) has multiple eigenvalues; and (3) if $\Delta<0$, the dynamical system (1) has a real eigenvalue and a pair of complex-conjugate eigenvalues.

The algebraic linear system solution (5), it is a starting point to analyze the equilibrium of the system, since both concepts are strongly related.

$$
A x+B=0 .
$$

The Rouché-Frobenius theorem uses an augmented matrix $(A \mid B)$, and provides the necessary conditions to establish the type of solution. The concept of equilibrium point and theorem are related as follows:
1. The linear system (5) has a unique solution, if and only if, the $\operatorname{rank}(A)=3$ and $\operatorname{rank}(A \mid B)=3$. In other words, a unique solution exists if $A$ is not singular. Hence, the dynamical system (1) has a hyperbolic equilibrium point given by $x^{*}=-A^{-1} B$, and the eigenvalues of matrix $A$ have the nonzero real part. Therefore, the system (1) is called a hyperbolic linear system.

2. The linear system (5) has multiple solutions if the $\operatorname{rank}(A)=\operatorname{rank}(A \mid B)<3$, that means, the matrix $A$ is singular. Consequently, the dynamical system (1) has an infinite number of equilibrium points. Because of that the constant term $\delta=0$ and $\Delta<0$ in Eq. (3), the system is nonhyperbolic, since at least one of its eigenvalues has the real part equal zero.

3. Finally, the linear system (5) does not have a solution if the $\operatorname{rank}(A) \neq \operatorname{rank}(A \mid B)$, consequently, $\operatorname{det}(A)=0$. Thus, the dynamical system (1) does not have equilibrium, and it is nonhyperbolic since at least one of its eigenvalues has the real part equal zero.

The first statement from the aforementioned theorem is the main characteristic of a self-excited attractor since its basin of attraction intersects with the neighborhood of the equilibrium point. On the other hand, the last two statements are related to hidden attractors. That means its basin of attraction does not intersect with the neighborhoods of equilibria. It is important to mention that the systems with a stable equilibrium fulfill the first statement of the Rouché-Frobenius theorem, however, they are considered as hidden attractors. The previous theorem is considered along with this paper since we pretend to generate a chaotic attractor through the switching of nonhyperbolic linear systems.

\section{Switched chaotic system without equilib- rium}

This section introduces to a system without equilibrium from the switching of two nonhyperbolic linear systems. Each linear system $A_{i} x=B_{i}$ belongs to domain $\mathcal{D}_{i} \subseteq \mathbb{R}^{3}$, where the matrix $A$ must be singular.

Assume that the flow of the switching system is given by $x(t)=\phi\left(x_{0}\right)$ such that $t \geq 0$. Moreover, assume that the system is dissipative and generates an attractor. As a case study, consider the next switching system

$$
\dot{x}= \begin{cases}A_{1} x+B_{1}, & \text { if } x_{1} \geq 0 \\ A_{2} x+B_{2}, & \text { if } x_{1}<0\end{cases}
$$

The domain of the system is $\mathcal{D}_{1}$ when $x_{1} \geq 0$ and $\mathcal{D}_{2}$ when $x_{1}<0$. The states vector $x$ and the vectors $B_{1}$ and $B_{2}$ are 
defined in Eq. (1). Finally, the matrices $A_{1}$ and $A_{2}$ are given by

$$
A_{1}=\left(\begin{array}{rrr}
0 & 1 & 1 \\
0 & -p & q \\
0 & -q & -k
\end{array}\right), \quad A_{2}=\left(\begin{array}{rrr}
0 & 1 & 1 \\
0 & -p & q \\
0 & -q & k
\end{array}\right) .
$$

Both previous matrices have a column vector zero, thus, $\operatorname{det}\left(A_{1}\right)=\operatorname{det}\left(A_{2}\right)=0$. This leads to the third statement of Rouché-Frobenius theorem, if and only if, the elements of $B_{1}$ and $B_{2}$ are nonzero. Then, this guarantees that the system (6) does not have equilibrium.

To obtain a chaotic behavior, firstly, the solution of the initial value problem must be of an oscillatory nature through of the eigenvalues. The characteristic polynomials associated with $\mathcal{D}_{1}$ and $\mathcal{D}_{2}$ are shown in the Eq. (8); meanwhile, their eigenvalues are given in the Eq. (9).

$$
\begin{aligned}
g(\lambda) & =\lambda^{3}+(p+k) \lambda^{2}+\left(q^{2}+k p\right) \lambda, \Longrightarrow \mathcal{D}_{1} \\
g(\mu) & =\mu^{3}+(p-k) \mu^{2}+\left(q^{2}-k p\right) \mu, \Longrightarrow \mathcal{D}_{2} \\
\lambda_{1} & =0, \quad \lambda_{2,3}=\frac{-(p+k) \pm \sqrt{(p+k)^{2}-4\left(q^{2}+k p\right)}}{2} \\
\mu_{1} & =0, \quad \mu_{2,3}=\frac{(k-p) \pm \sqrt{(p-k)^{2}-4\left(q^{2}-k p\right)}}{2} .
\end{aligned}
$$

Note that these matrices have an eigenvalue equal to zero because $g(\lambda)$ and $g(\mu)$ do not have independent terms. Thus, the solution set is of oscillatory nature if the last two eigenvalues are one pair of complex-conjugates, that is to say, $(p+k)^{2}<4\left(q^{2}+k p\right)$ and $(p-k)^{2}<4\left(q^{2}-k p\right)$, for $\mathcal{D}_{1}$ and $\mathcal{D}_{2}$, respectively. Therefore, according to Eq. (4) the discriminant is calculated as $\Delta=\beta^{2} \gamma^{2}-4 \gamma^{3}<0$.

Let $p=0.25$ and $q=3$ be the elements of matrices $A_{1}$ and $A_{2}$, and let $b_{1}=b_{2}=0$ and $b_{3}=0.05$ be elements of vectors $B_{1}$ and $B_{2}$. Taking into account the above inequalities, the parameter $k$ is defined through a systematic search assisted by software based on the methods proposed in Ref. [39]. This search consists in to find both the value of $k$ as the initial conditions, such that the Lyapunov exponents or the bifurcation diagrams show the presence of chaos in the system.

In this way, the system (6) has chaotic behavior when the parameter $k=2.3$ and the initial conditions are $\left(x_{1_{0}}, x_{2_{0}}, x_{3_{0}}\right)=(0.05,0.05,0.05)$. The properties of the characteristic polynomials of the domains $\mathcal{D}_{1}$ and $\mathcal{D}_{2}$ were verified. In the domain $\mathcal{D}_{1}$, the discriminant is $\Delta=$ -2915.21 and the eigenvalues associated are $\lambda_{1}=0$ and $\lambda_{2,3}=-1.275 \pm 2.8195 i$. Meanwhile, in the domain $\mathcal{D}_{2}$, the discriminant is $\Delta=-2093.75$ and the eigenvalues are $\mu_{1}=0$ and $\mu_{2,3}=1.025 \pm 2.7156 i$.

The Lyapunov exponents are denoted by $\lambda_{L_{1}}=0.1086$, $\lambda_{L_{2}}=0$ and $\lambda_{L_{3}}=-0.3509$, and the Kaplan-Yorke dimension is $D_{K Y}=2.3065$, which indicate that there exist a strange attractor. The sum of Lyapunov exponents $\lambda_{L_{1}}+\lambda_{L_{2}}+\lambda_{L_{3}}=-0.2423$ indicates the system is dissipative. However, the dissipation of each domain is calculated by the variation of a small element of volume $V(t)$ as
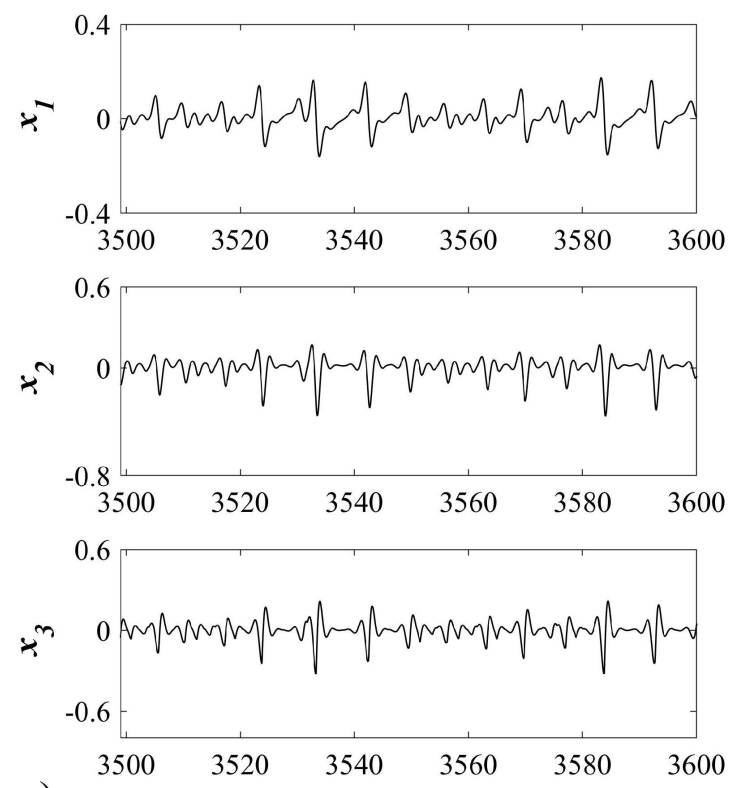

a)

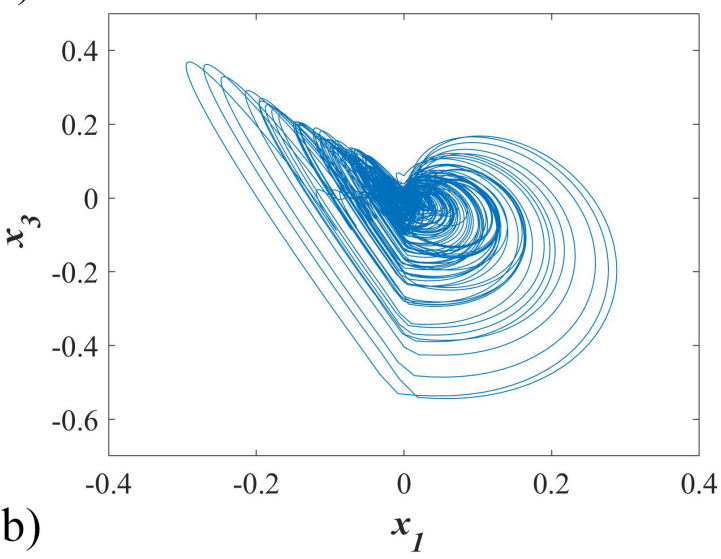

FIgURE 1. a) Time series of states of the switching system without equilibrium. b) Phase portrait of the chaotic attractor in the switching system without equilibrium on $x_{1}-x_{3}$-plane.

$$
\begin{aligned}
\nabla V(t) & =\frac{\partial \dot{x}_{1}}{\partial x_{1}}+\frac{\partial \dot{x}_{2}}{\partial x_{2}}+\frac{\partial \dot{x}_{3}}{\partial x_{3}} \\
& =\left\{\begin{array}{rll}
2.05, & \text { for } & \mathcal{D}_{1} \\
-2.55, & \text { for } & \mathcal{D}_{2}
\end{array}\right.
\end{aligned}
$$

In Fig. 1, it is can observe the time series of states $x_{1}, x_{2}$, and $x_{3}$. Also, the hidden attractor generated from the switching system is shown. The frequency spectra of the time series confirm that they are of a chaotic nature in Fig. 2.

\section{A switched chaotic system with an infinite number of equilibrium points.}

This section presents the switching of a linear system with an infinite number of equilibria and a linear system without 

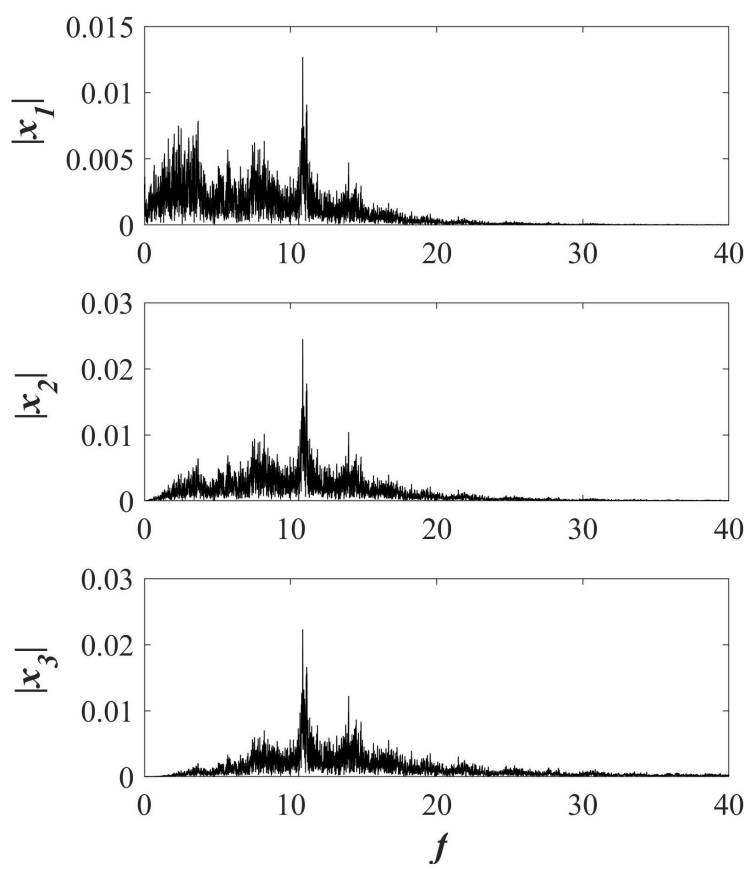

FIGURE 2. Frequency spectra generated from the time series of the switching system without equilibrium.

equilibrium. Hence, the equilibrium of the switched dynamical system is an infinite number of equilibrium points. Consider the next system

$$
\dot{x}=\left\{\begin{array}{lll}
A_{3} x+B_{3}, & \text { if } & x_{1} \geq 0 \\
A_{4} x+B_{4}, & \text { if } & x_{1}<0
\end{array}\right.
$$

where $x=\left(x_{1}, x_{2}, x_{3}\right)^{T}$ is the states vector, and the matrices are denoted by

$$
\begin{aligned}
A_{3} & =\left(\begin{array}{rrr}
0 & 1 & 1 \\
0 & -p & q \\
0 & -k_{1} & k_{3}
\end{array}\right), \\
A_{4} & =\left(\begin{array}{rrr}
0 & 1 & 1 \\
0 & p & q \\
0 & -k_{2} & -k_{3}
\end{array}\right) .
\end{aligned}
$$

Both matrices have $\operatorname{det}\left(A_{3}\right)=\operatorname{det}\left(A_{4}\right)=0$. The domain of system (11) is represented by $\mathcal{D}_{3}$ when $x_{1} \geq 0$ and $\mathcal{D}_{4}$ when $x_{1}<0$. The domain $\mathcal{D}_{3}$ has an infinite number of equilibrium points, if and only if, $B_{3}=(0,0,0)^{T}$. This leads to the second statement of Rouché-Frobenius. The equilibrium of $\mathcal{D}_{3}$ has been obtained when the following algebraic equations are solved:

$$
\begin{aligned}
x_{2}+x_{3} & =0 \\
-p x_{2}+q x_{3} & =0 \\
-k_{1} x_{2}+k_{3} x_{3} & =0 .
\end{aligned}
$$

Thus, the equilibrium is defined by $E\left(x_{1}^{*}, 0,0\right)$. On the other hand, the domain $\mathcal{D}_{4}$ does not have equilibrium, since the vector $B_{4}=\left(0,0, b_{3}\right)^{T}$ with $b_{3} \neq 0$. Therefore, combining the systems of the domains $\mathcal{D}_{3}$ and $\mathcal{D}_{4}$ the switched system (11) has an infinite number of equilibrium points.

According to equation (3), the characteristic polynomials of the matrices in (12) are as follows

$$
\begin{aligned}
& g(\lambda)=\lambda^{3}+\left(p-k_{3}\right) \lambda^{2}+\left(k_{1} q-k_{3} p\right) \lambda, \Longrightarrow \mathcal{D}_{3} \\
& g(\mu)=\mu^{3}+\left(k_{3}-p\right) \mu^{2}+\left(k_{2} q-k_{3} p\right) \mu, \Longrightarrow \mathcal{D}_{4} .
\end{aligned}
$$

Then the discriminant of $g(\lambda)$ and $g(\mu)$ is $\Delta=\beta^{2} \gamma^{2}-4 \gamma^{3}$. Thus, their corresponding eigenvalues are defined by

$$
\begin{aligned}
\lambda_{1} & =0 \\
\lambda_{2,3} & =\frac{\left(k_{3}-p\right)}{2} \pm \frac{\sqrt{\left(p-k_{3}\right)^{2}-4\left(k_{1} q-k_{3} p\right)}}{2} \\
\mu_{1} & =0 \\
\mu_{2,3} & =\frac{\left(p-k_{3}\right)}{2} \pm \frac{\sqrt{\left(k_{3}-p\right)^{2}-4\left(k_{2} q-k_{3} p\right)}}{2} .
\end{aligned}
$$

Like in the previous section, domains should be governed by eigenvalues such as unstable focus or stable focus. This provides an oscillatory solution to the initial value problem. This implies the next inequalities from Eq. (15) $\left(k_{3}-p\right)^{2}<$ $4\left(k_{1} q-k_{3} p\right)$ and $\left(k_{3}-p\right)^{2}<4\left(k_{2} q-k_{3} p\right)$ in $\mathcal{D}_{3}$ and $\mathcal{D}_{4}$, respectively.

Assume that the parameters of the system (11) are $p=$ $0.25, q=3, b_{3}=0.2$, and $k_{3}=1.75$. According to the inequalities aforementioned, we have an unstable focus and a stable focus when the parameters $k_{1}>(1 / 3)$ and $k_{2}>(1 / 3)$, respectively. In the same way, the initial conditions and the parameters $k_{1}$ and $k_{2}$ are found through the systematic search by software. Chaotic behavior was found when $k_{1}=1.9$ and $k_{2}=4.1$ with initial conditions $\left(x_{1_{0}}, x_{2_{0}}, x_{3_{0}}\right)=(0.05,0.05,0.05)$. The eigenvalues of domain $\mathcal{D}_{3}$ are $\lambda_{1}=0$ and $\lambda_{2,3}=0.75 \pm 2.1679 i$ with $\Delta=-520.645$. On the domain $\mathcal{D}_{4}$ their eigenvalues are $\mu_{1}=0$ and $\mu_{2,3}=-0.75 \pm 3.3615 i$ with $\Delta=-6360.49$.

The Lyapunov exponents of the switched system with an infinite number of equilibrium points are $\lambda_{L_{1}}=0.04298$, $\lambda_{L_{2}}=0$ and $\lambda_{L_{3}}=-0.1129$, and the Kaplan -Yorke dimension is $D_{K Y}=2.38070$. This confirms the presence of chaos and the existence of a strange attractor. This system is dissipative since the sum of the Lyapunov exponents is negative, we have $\lambda_{L_{1}}+\lambda_{L_{2}}+\lambda_{L_{3}}=-0.0631$. Meanwhile, the divergence and convergence of the flow of each domain are obtained in Eq. (16). It is clear that domain $\mathcal{D}_{4}$ is dissipative, but $\mathcal{D}_{3}$ is not.

$$
\begin{aligned}
\nabla V(t) & =\frac{\partial \dot{x}_{1}}{\partial x_{1}}+\frac{\partial \dot{x}_{2}}{\partial x_{2}}+\frac{\partial \dot{x}_{3}}{\partial x_{3}} \\
& =\left\{\begin{array}{rll}
1.5, & \text { for } & \mathcal{D}_{3} \\
-1.5, & \text { for } & \mathcal{D}_{4}
\end{array}\right.
\end{aligned}
$$

Figure 3 shows a section of the times series of states $x_{1}, x_{2}$ and $x_{3}$, moreover, the phase portrait of the hidden attractor on $x_{1}-x_{3}$ plane is present. The frequency spectra for each time series are illustrated in Fig. 4. 

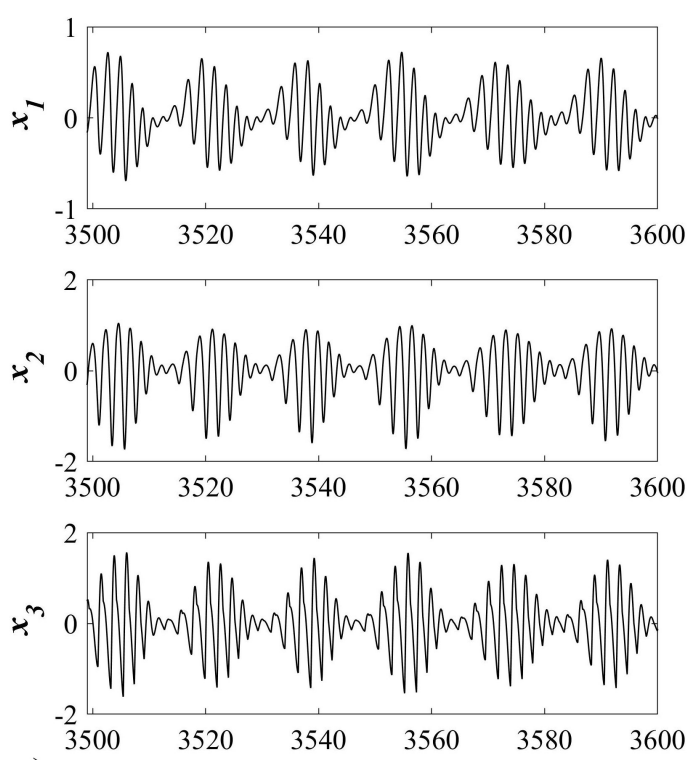

a)

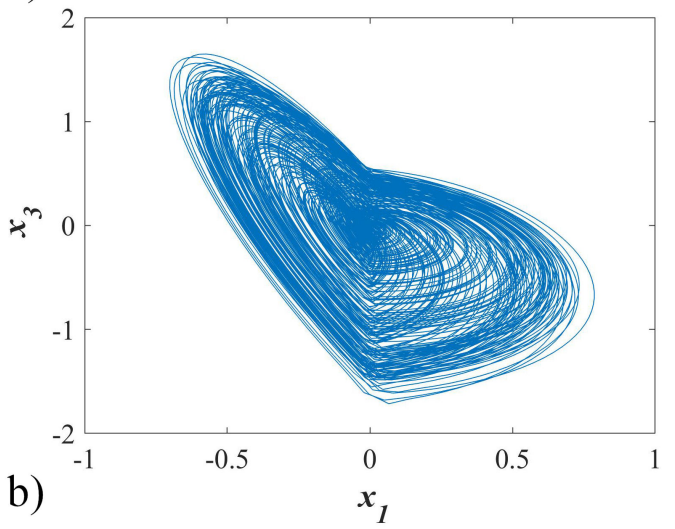

FIgURE 3. a) Time series of states of the switching system with an infinite number of equilibrium points. b) Phase portrait of the chaotic attractor in switching system with an infinite number of equilibrium points on $x_{1}-x_{3}$-plane.

\section{Switched chaotic system with only one sta- ble equilibrium point.}

This system is developed by the switching of a linear system without equilibrium and a linear system with one stable equilibrium. To analyze this case, a switching system is proposed as follows

$$
\dot{x}=\left\{\begin{array}{lll}
A_{5} x+B_{5}, & \text { if } \quad x_{1} \geq 0 \\
A_{6} x+B_{6}, & \text { if } \quad x_{1}<0
\end{array}\right.
$$

where the matrices $A_{5}$ and $A_{6}$ are given by

$$
\begin{gathered}
A_{5}=\left(\begin{array}{rrr}
0 & 1 & 1 \\
0 & -p & q \\
0 & -q & k_{1}
\end{array}\right), \\
A_{6}=\left(\begin{array}{rrr}
0 & 1 & 1 \\
0 & -p & q \\
-k_{2} & -q & -k_{1}
\end{array}\right),
\end{gathered}
$$
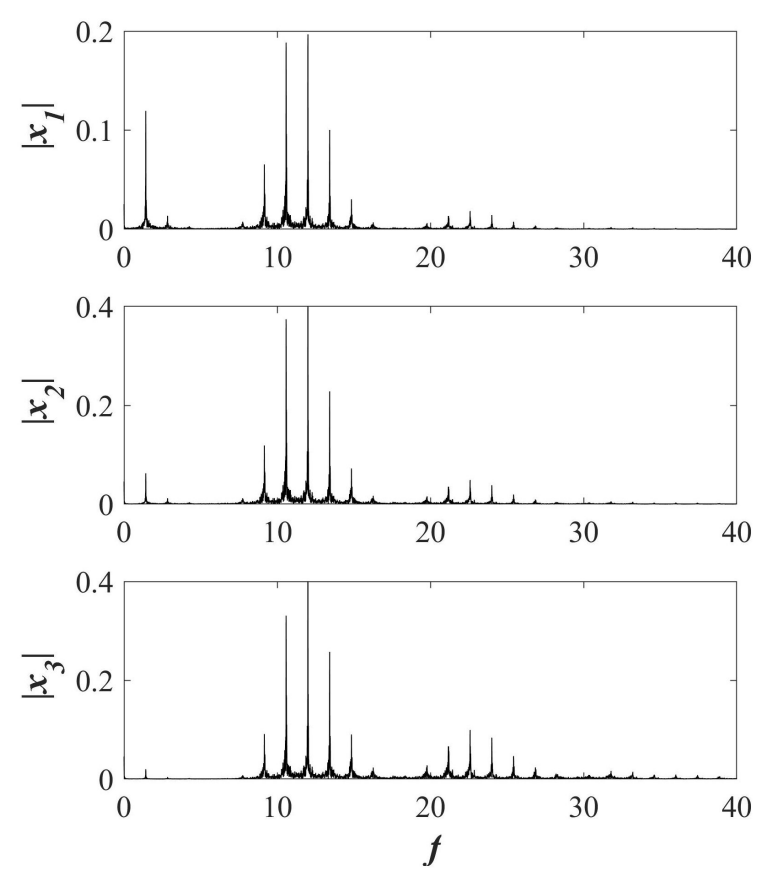

FIGURE 4. Frequency spectra generated from the time series of the switching system with an infinite number of equilibrium points.

and the values of the parameters $p$ and $q$ are the same as the previous sections.

The linear system $A_{5} x+B_{5}$ does not have an equilibrium, if and only if, $B_{5}=\left(0,0, b_{3}\right)^{T}$, with $b_{3} \neq 0$. This system was studied in detail in Sec. 3 as well as their eigenvalues. On the other hand, the system $A_{6} x+B_{6}$ with $B_{6}=(0,0,0)^{T}$ has one equilibrium point when the next algebraic system is solved,

$$
\begin{array}{r}
x_{2}+x_{3}=0 \\
-p x_{2}+q x_{3}=0 \\
-k_{2} x_{1}-q x_{2}-k_{1} x_{3}=0 .
\end{array}
$$

Thus, this linear system has one equilibrium point in $E(0,0,0)$. This equilibrium must be stable, which means, all the eigenvalues associated with the matrix $A_{6}$ must have a negative real part. Therefore the system (17) has one equilibrium point.

Now, the analysis of the matrix $A_{6}$ is carried out from the characteristic polynomial

$$
g_{6}(\lambda)=\lambda^{3}+\left(k_{1}+p\right) \lambda^{2}+\left(k_{1} p+q^{2}+k_{2}\right) \lambda+k_{2}(p+q) .
$$

This polynomial has an independent term, that means, the determinant of the matrix $A_{6}$ is nonzero. Thus, Cardano's method is used to solve the polynomial. Consider the next cubic equation

$$
g_{6}(\lambda)=\lambda^{3}+a \lambda^{2}+b \lambda+c=0,
$$


where parameters are $a=\left(k_{1}+p\right), b=\left(k_{1} p+q^{2}+k_{2}\right)$ and $c=k_{2}(p+q)$. Now, we perform a change of variable as follows:

$$
\lambda=y-\frac{a}{3},
$$

and substituting in Eq. (21) the quadratic term is removed. Hence, the reduced cubic equation is obtained

$$
y^{3}+u y+v=0,
$$

where

$$
\begin{aligned}
u & =\frac{3 b-a^{2}}{3}, \\
v & =\frac{2 a^{3}-9 a b+27 c}{27}, \\
\sigma & =\left(\frac{v}{2}\right)^{2}+\left(\frac{u}{3}\right)^{3} .
\end{aligned}
$$

The cubic Eq. (23) has three roots, and they are calculated as follows:

$$
\begin{aligned}
& y_{1}=P+Q \\
& y_{2}=-\frac{1}{2}(P+Q)+i \frac{\sqrt{3}}{2}(P-Q) \\
& y_{3}=-\frac{1}{2}(P+Q)-i \frac{\sqrt{3}}{2}(P-Q),
\end{aligned}
$$

where the values of parameters $P$ y $Q$ are defined as

$$
\begin{aligned}
& P=\sqrt[3]{-\frac{v}{2}+\sqrt{\sigma}} \\
& Q=\sqrt[3]{-\frac{v}{2}-\sqrt{\sigma}} .
\end{aligned}
$$

Finally, the roots of Eq. (21) are calculated from the solutions of Eq. (23). Consequently, we have

$$
\lambda_{i}=y_{i}-\frac{a}{3} .
$$

Notice that the parameter $\sigma$ is equivalent to the discriminant in Eq. (4). Then, the matrix $A_{6}$ has a real eigenvalue $\lambda_{1}$ and a pair of complex-conjugate eigenvalues $\lambda_{2,3}$ if $\sigma>0$.

Taking into account the mathematical expressions above, the values of the parameters $k_{1}$ and $k_{2}$ are found, by a systematic search, such that all eigenvalues have the negative real part. The system (17) is chaotic and has an equilibrium stable when $k_{1}=2$ and $k_{2}=2.6$, with the initial conditions $\left(x_{1_{0}}, x_{2_{0}}, x_{3_{0}}\right)=(0.05,0.05,0.05)$. Analyzing the local behavior in $x_{1} \geq 0$, the eigenvalues of $g_{5}(\mu)$ (it was studied in detail in Sec. 3) are $\mu_{1}=0$ and $\mu_{2,3}=0.875 \pm 2.7811 i$ with $\Delta=-2235.23$. Hence, in $x_{1}<0$, the eigenvalues of $g_{6}(\lambda)$ are $\lambda_{1}=-0.7710$ and $\lambda_{2,3}=-0.7395 \pm 3.2270 i$ with $\Delta=-4516.99$.

This system is chaotic because the Lyapunov exponents are $\lambda_{L_{1}}=0.3562, \lambda_{L_{2}}=0$ and $\lambda_{L_{3}}=-0.5560$, and the

\begin{tabular}{|c|c|c|c|c|c|}
\hline Case & Parameters & Equilibrium & Eigenvalues & LEs & $\mathrm{D}_{\mathrm{KY}}$ \\
\hline$S W_{N E}$ & $k=3.11$ & None & $\begin{aligned} \mu_{1} & =0 \\
\mu_{2,3} & =-1.68 \pm 2.6372 i \\
\lambda_{1} & =0 \\
\lambda_{2,3} & =1.43 \pm 2.4854 i\end{aligned}$ & $\begin{array}{l}\lambda_{L_{1}}=0.1214 \\
\lambda_{L_{2}}=0 \\
\lambda_{L_{3}}=-0.7168\end{array}$ & 2.1682 \\
\hline$S W_{L E}$ & $\begin{array}{l}k_{1}=2 \\
k_{2}=4.1 \\
k_{3}=1.75\end{array}$ & $E\left(x^{*}, 0,0\right)$ & $\begin{aligned} \mu_{1} & =0 \\
\mu_{2,3} & =0.75 \pm 2.2360 i \\
\lambda_{1} & =0 \\
\lambda_{2,3} & =-0.75 \pm 3.3615 i\end{aligned}$ & $\begin{array}{l}\lambda_{L_{1}}=0.0527 \\
\lambda_{L_{2}}=0 \\
\lambda_{L_{3}}=-0.0939\end{array}$ & 2.5611 \\
\hline$S W_{S E}$ & $\begin{array}{l}k_{1}=2 \\
k_{2}=4\end{array}$ & $E(0,0,0)$ & $\begin{aligned} \mu_{1} & =0 \\
\mu_{2,3} & =0.875 \pm 2.7810 i \\
\lambda_{1} & =-0.9371 \\
\lambda_{2,3} & =-0.4064 \pm 3.4019 i\end{aligned}$ & $\begin{array}{l}\lambda_{L_{1}}=0.4849 \\
\lambda_{L_{2}}=0 \\
\lambda_{L_{3}}=-0.8892\end{array}$ & 2.5374 \\
\hline
\end{tabular}
existence of the strange attractor is confirmed by the KaplanYorke dimension, $D_{K Y}=2.6548$. Besides the system dissipation is verified by the sum of the system of the Lyapunov exponents is negative and is $\lambda_{L_{1}}+\lambda_{L_{2}}+\lambda_{L_{3}}=-0.1998$. Thus, the divergence of the flow of each domain is

$$
\nabla V(t)=\frac{\partial \dot{x}_{1}}{\partial x_{1}}+\frac{\partial \dot{x}_{2}}{\partial x_{2}}+\frac{\partial \dot{x}_{3}}{\partial x_{3}}=\left\{\begin{aligned}
1.75, & \mathcal{D}_{3} \\
-2.25, & \mathcal{D}_{4} .
\end{aligned}\right.
$$

The time series and chaotic attractor are presented in Fig. 5. As can be seen in Fig. 6, frequency spectra indicate the chaoticity of the system. 

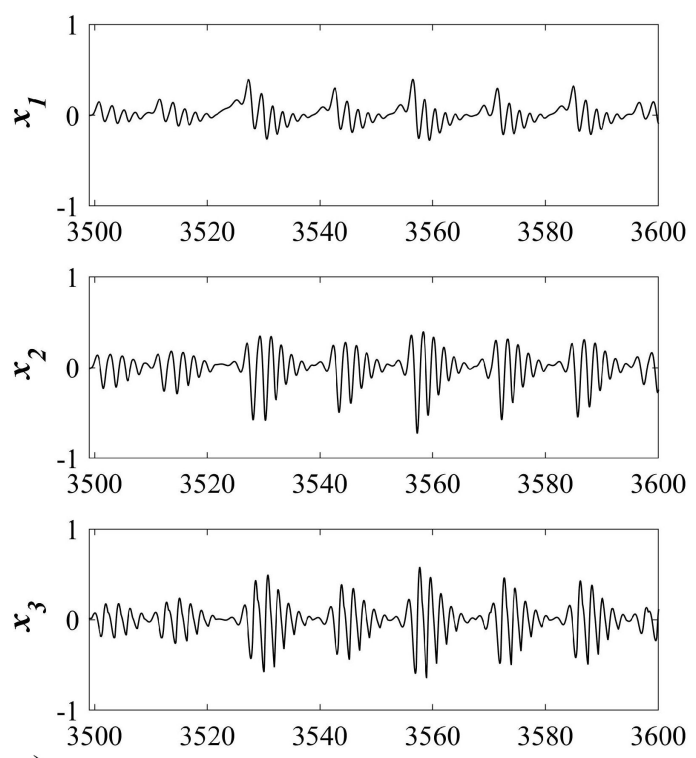

a) $\boldsymbol{t}$

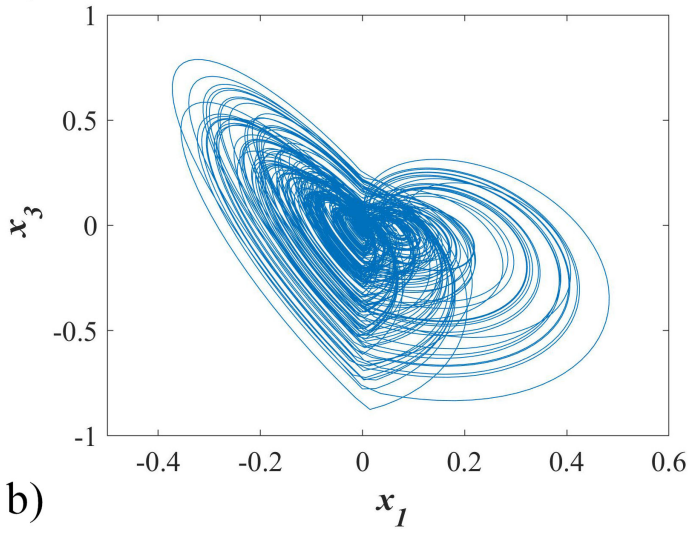

FIgURE 5. a) Time series of states of the switching system with only one stable equilibrium point. b) Phase portrait of the chaotic attractor in a switching system with only one stable equilibrium point on $x_{1}-x_{3}$-plane.

\section{Discussion}

The dynamic of the previous systems was analyzed through the parameter variation found by the systematic search. For the switching system without equilibrium, the parameter $k$ was varied in the interval from 2 to 5 . Figure 7 shows the system dynamics through the Lyapunov exponents. As can be observed, the chaoticity exists in the system for some values into the intervals from and 2.1 to 2.5 and from 2.9 to 3.2 .

For the switching system with an infinite number of equilibrium points, its dynamic has been plotted in a Lyapunov exponents diagram (Fig. 8) concerning parameter $k_{1}$ when is varied from 1.5 to 5 . It can be observed a small region of chaos from 1.6 to 2.2 , though limit cycles exist in the interval from 2.2 to 5 . However, note that the system power spectrum presented in Sec. 4 (Fig. 4) shows strong amplitude peaks. Also, the Lyapunov exponents are very small, and its almost impossible to distinguish from zero, this indicates the strong probability that the system with an infinite
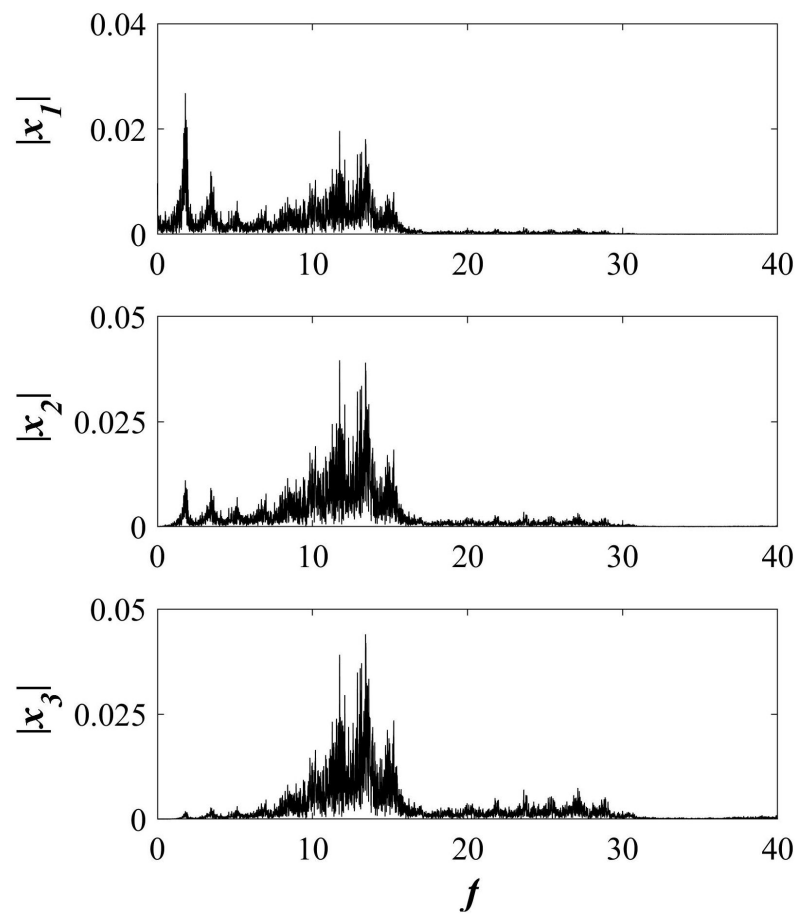

FIGURE 6. Frequency spectra generated from the time series of the switching system with only one stable equilibrium point.

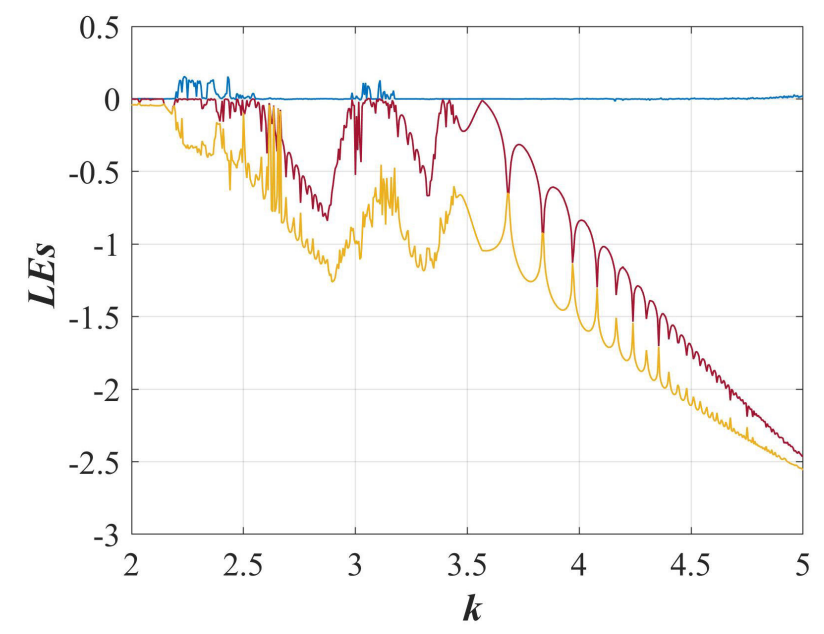

FIGURE 7. Dynamic of the Lyapunov exponents of the switching system without equilibrium when parameter $k$ is varied from 2 to 5 .

of equilibrium points may be a quasi-periodic. In this way, not only can beautiful chaotic systems can be obtained by mixing linear systems, but even quasi-periodic dynamics can be obtained as well.

Similarly, a Lyapunov exponents diagram has been plotted in Fig. 9 to the switching system with only one stable equilibrium, where the parameter $k_{2}$ was varied from 0 to 4.5 . It can be appreciated that the region to $k_{2}>2.5$, some values indicate chaos in the system.

It is hard work to find chaotic behavior in a switching system, and generate hidden attractor since they are developed from the combination of linear systems no-solution, multiple 


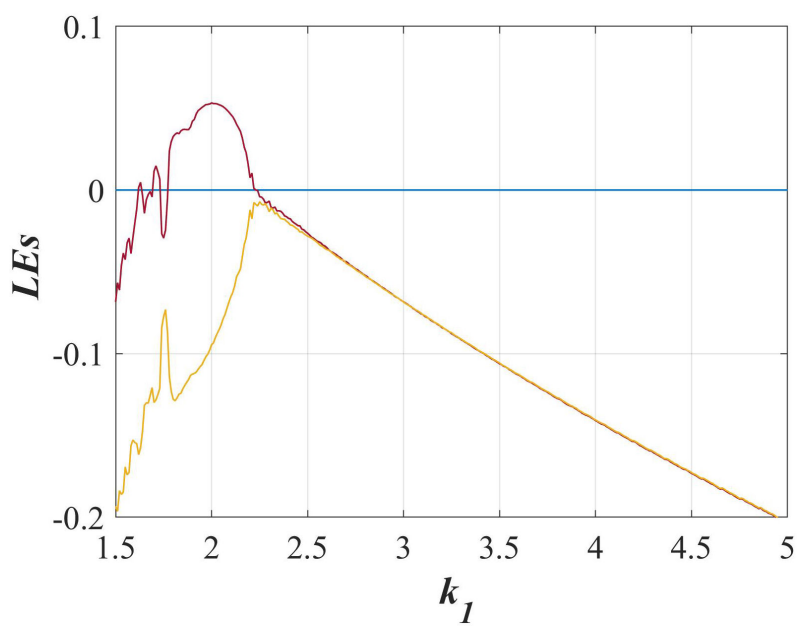

FIGURE 8. Dynamic of the Lyapunov exponents of the switching system with an infinite number of equilibria when $k_{2}=4.1$ and $k_{1}$ is varied from 1.5 to 5 .

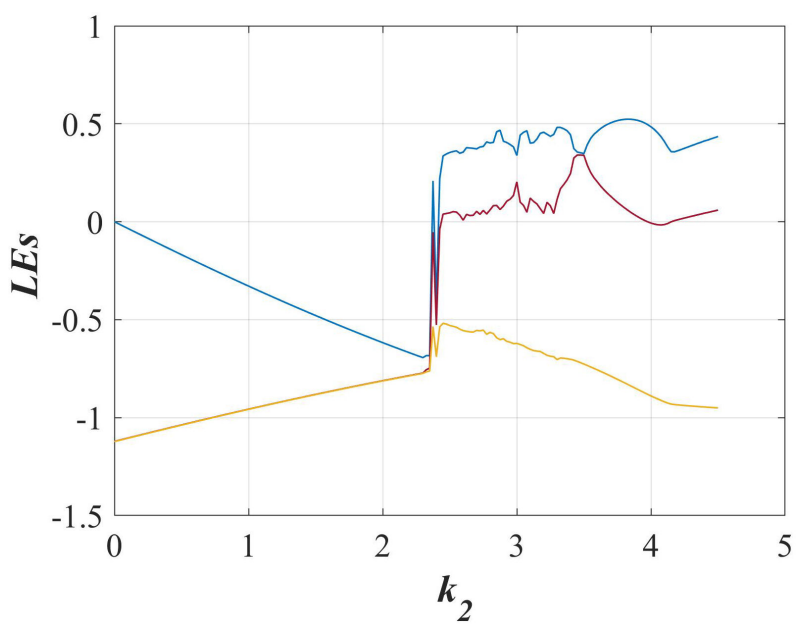

FIGURE 9. Dynamic of the Lyapunov exponents of the switching system with only one stable equilibrium when $k_{1}=2$ and $k_{2}$ is varied from 0 to 4.5 .

solutions, or a unique solution when $\dot{x}=0$. Besides the presence of an eigenvalue equal to zero and the absence of a saddle eigenvalue, the switched systems without equilibrium, and switched systems with an infinite number of equilibria depend only on eigenvalues of stable focus and unstable focus to obtain an oscillatory solution. In other words, the system oscillates towards infinity, and through switching, the system oscillates towards stability. The above does not ensure that the system is chaotic, but it is too difficult to control the orbits of the system flow. On the other hand, all the eigenvalues in the systems with only one stable equilibrium have a negative real part. This indicates that the linear system is stable or asymptotically stable. For this reason, it was decided to include a system without equilibrium with its eigenvalues with a positive real part, so that the system orbits would expand and contract.

Despite this, we have found that the dynamics of these systems are bounded and converges towards the attractor for a wide range of initial conditions and different system parameters. Table I summarized some cases of the switching systems above (with different values of their parameters), where chaos is present. The system without equilibrium is stood for $S W_{N E}$, the system with many equilibria as $S W_{L E}$ and the system with one stable equilibrium as $S W_{S E}$.

The above dynamics systems were numerically solved by the fifth-order Runge-Kutta method. The simulations were generated in a time interval from 0 to 5000 with an integration step $\mathrm{dt}=0.001$. On the other hand, the systematic search mentioned in each section above is based on the methods proposed in the reference [39]. This search consists in to find both the values of some parameters as the initial conditions, such that the Lyapunov exponents show the presence of chaos in the system. The Lyapunov exponents were calculated using the algorithm of Wolf [40], and they were verified using the algorithm from the reference [41].

\section{Conclusions}

A family of switching systems that generates hidden attractors is proposed in this paper. The presence of stable equilibrium, an infinite number of equilibria, and no-equilibrium is in this family. The development of these switching systems is based on the linear systems theory. It is important to mention that most of the hidden attractors reported in the literature are constructed by systems with quadratic nonlinearities. However, a few hidden attractors constructed through the switching of linear systems have been found in the literature. It would be interesting to implement these systems on chaos-based applications.

\section{Acknowledgements}

F. Delgado-Aranda is a doctoral fellow of the CONACYT in the Graduate Program on Applied Science at IICO-UASLP acknowledges the CONACYT financial support through grant No. 419416. The authors would like to thank the reviewers for their valuable comments and suggestions on the article.
1. E. N. Lorenz, J. Atmos. Sci. 20 (1963) 130. https: //doi.org/10.1175/1520-0469(1963)020<0130: $\mathrm{DNF}\rangle 2.0 . \mathrm{CO} ; 2$

2. C. Li, Y. Liu, T. Xie, Z. Q. Chen Michael, Nonlin- ear Dyn. 73 (2013) 2083. https://doi.org/10.1007/ s11071-013-0924-6

3. K. Z. Li, M. C. Zhao, X. C. Fu, IEEE Trans. Circuits Syst. I 56 (2009) 2280. https://doi.org/10.1063/1.3622678 
4. I. M. Ginarsa, A. Soeprijanto, M. H. Purnomo, Int. J. Electr. Power Energy Syst. 46 (2013) 79. https : / doi.org/10. $1016 / j$.ijepes.2012.10.005

5. G. Chen, T. Ueta, Int. J. Bifurcat. Chaos 9 (1999) 1465. https://doi.org/10.1142/S0218127499001024

6. J. Lü, G. Chen, Int. J. Bifurcat. Chaos 12 (2002) 659. https : //doi.org/10.1142/S0218127402004620

7. L. O. Chua, M. Komuro, T. Matsumoto, IEEE T. Circuits Syst. 33 (1986) 1072. DOI : 10.1109/TCS . 1986.1085869

8. O. E. Rössler, Phys. Lett. 57A (1976) 397. https://doi. org/10.1016/0375-9601 (76)90101-8

9. J. C. Sprott, Phys Rev. E50 (1994) 647. https : //doi . org/ 10.1103/PhysRevE.50.R647

10. J. Lü, G. Chen, Int. J. Bifurcat. Chaos 16 (2006) 775. https : //doi.org/10.1142/S0218127406015179

11. J. Lü, X. Yu, G. Chen, IEEE Trans. Circuits Syst. I 50 (2003) 198. DOI:10.1109/TCSI.2004.838151

12. E. Campos-Cantón, J. G. Barajas-Ramírez, G. Solís-Perales, R. Femat, Chaos 20 (2010) 013116. https: / / doi.org/10. $1063 / 1.3314278$

13. O. E. Rössler, Phys. Lett. 57A (1979) 155. https://doi. org/10.1016/0375-9601(79) 90150-6

14. V.T. Pham, C. Volos, T. Kapitaniak, Systems with hidden attractors: From theory to realization in circuits (Springer, Switzerland, 2017).

15. L. P. Shilnikov, A. Shilnikov, D. Turaev, L. O. Chua, Methods of qualitative theory in nonlinear dynamics (World Scientific, Singapore 1998).

16. L. P. Shilnikov, Sov. Math. Docklady 6 (1965) 163.

17. V.T. Pham, C. Volos, S. Jafari, Z. Wei, X. Wang, Int. J. Bifurcat. Chaos 24 (2014) 1450073. https: //doi .org/10.1142/ S0218127414500734

18. C. Wang, C. Hu, J. Han, S. Cang, Adv. Math. Phys. (2016) 2016.

19. A. T. Azar et al., Complexity (2017) 2017. https://doi. org/10.1155/2017/7871467

20. Z. Wei, Phys. Lett. A 376 (2011) 102. https://doi.org/ $10.1016 / j \cdot$ physleta.2011.10.040

21. S. Jafari, J. C. Sprott, S. M. R. H. Golpayegani, Phys. Lett. A 377 (2013) 699. https: / doi.org/10.1016/j. physleta.2013.01.009

22. Z. Wang, S. Cang, E. O. Ochola, Y. Sun, Nonlinear Dyn 69 (2012) 531. https://doi.org/10.1007/ s11071-011-0284-z

23. X. Hu, C. Liu, L. Liu, J. Ni, S. Ki, Nonlinear Dyn 86 (2016) 1725 .
24. S. Jafari, V. T. Pham, T. Kapitaniak, Int. J. Bifurcat. Chaos 26 (2016). https://doi.org/10.1142/ S0218127416500310

25. D. R. Tahir, S. Jafari, V. T. Pham, C. Volos, X. Wang, Int. J. Bifurcat. Chaos 25 (2015) 1550. https://doi.org/10. 1142/S021812741550056X

26. H. Li, X. Liao, M. Luo, Nonlinear Dyn 68 (2012) 137. https: //doi.org/10.1007/s11071-011-0210-4

27. D. Cafagna, G. Grassi, Commun. Nonlinear Sci. Numer. Simul. 19 (2014). https://doi.org/10.1016/j. cnsns.2014.02.017

28. S. Jafari, J. C. Sprott, Chaos Soliton Fract. 57 (2013) 79. https://doi.org/10.1016/j.chaos.2013.08. 018

29. L. Chunbiao, J. C. Sprott, Phys. Lett. A 378 (2014) 178. https://doi.org/10.1016/j.physleta.2013. 11.004

30. Q. Li, S. Hu, S. Tang, G. Zeng, Int. J. Circ. Theor. Appl. 42 (2014) 1172.https://doi.org/10.1002/cta.1912

31. P. Zhou, F. Yang, Nonlinear Dyn 76 (2014) 473. https: //doi.org/10.1007/s11071-013-1140-0

32. P. Zhou, K. Huang, C. Yang, Discrete Dyn. Nat. Soc. (2013) 2013.

33. X. Wang, G. Chen, Commun. Nonlinear Sci. Numer. Simul. 17 (2012) 1264. https://doi.org/10.1016/j.cnsns. 2011.07 .017

34. V. T. Pham, S. Jafari, C. Volos, T. Kapitaniak, Int J. Bifurc. Chaos 27 (2017) 1750138.

35. Q. Yang, G. Chen, Int J. Bifurc. Chaos 18, (2008) 1393. https://doi.org/10.1142/S0218127408021063

36. Q. Yang, Z. Wei, G. Chen, Int J. Bifurc. Chaos 20 (2010) 1061.

37. M. R. Jeffrey, Int J. Bifurc. Chaos 26 (2016) 1650087. https: //doi.org/10.1142/S0218127416500875

38. G. A. Leonov, N. V. Kuznetsov, Int J. Bifurc. Chaos 23 (2013) 1330002-1. https://doi.org/10.1016/j. ifacol.2017.08.470

39. J. C. Sprott, Elegant chaos: algebraically simple chaotic flows (World Scientific 2010).

40. A. Wolf, J. B. Swift, H. L. Swinney, J. A. Vastano, Physica D 16 (1985) 285. https://doi.org/10.1016/ 0167-2789(85) 90011-9

41. M. F. Danca, N. Kuznetsov, Int J. Bifurc. Chaos 28 (2018) 1850067. https://doi.org/10.1142/ S0218127418500670 